\title{
Investigation of Clinical Factors Related To Diabetic Vascular Endothelial Function Using Reactive Hyperemia Peripheral Arterial Tonometry
}

\author{
Shunsuke Kikuchi, Junnosuke Miura*, Kanako Shimura, Tetsuya Babazono And Yasuko Uchigata \\ Diabetes Center, Tokyo Women's Medical University School of Medicine, Japan
}

\author{
Received: June 18, 2018; Accepted: July 02,2018; Published: July 05,2018 \\ *Corresponding author: Junnosuke Miura, Diabetes Center, Tokyo Women's Medical University School of Medicine, Tokyo 162-8666, Japan, Tel: +81-3-3353- \\ 8111; Fax: +81-3-3358-1941; E-mail:jmiura.dmc@twmu.ac.jp
}

\begin{abstract}
Background: Vascular endothelial function can be evaluated based on reactive hyperemia peripheral arterial tonometry (RH-PAT). This information can be used to perform appropriate risk assessments and therapeutic interventions. We investigated the relationship between the RHPAT index (RHI) and cardiovascular risk factors or daily blood glucose variations according to type of diabetes.

Methods: This study included 25 patients with diabetes but no severe organ damage (type 1 diabetes [T1D]: 12 cases, type 2 diabetes [T2D]: 13 cases). The patients were evaluated to determine their RHI and its relation with continuous glucose monitoring (CGM) parameters, glycated hemoglobin (HbA1c), glycoalbumin (GA), high-density lipoprotein-cholesterol (HDL-C), triglycerides (TG), and tumor necrosis factor (TNF- $\alpha$ ). Various other clinical and laboratory parameters were analyzed for associations with RHI.

Results: Mean ages were $40 \pm 12$ years in the T1D group and $54 \pm 13$ years in the T2D group. Mean HbA1c levels were $7.6 \pm 1.0 \%$ in the T1D group and $7.0 \pm 0.9 \%$ in the T2D group. RHI was positively correlated with GA among all cases $(p=0.02)$ and among T1D cases $(p=0.011)$. Among T2D cases, RHI was positively correlated with HDL-C $(p=0.034)$ and negatively correlated with TG $(p=0.03)$ and TNF- $\alpha(p=0.047)$. In the multivariate analysis, RHI was independently correlated with GA among all cases $(\beta=0.471, p=0.02)$ and among T1D cases $(\beta=0.727$, $p=0.011)$. RHI was also independently correlated with HDL-C among T2D cases $(\beta=0.586, p=0.045)$. Among T1D cases, GA was negatively correlated with hypoglycemia frequency $(\mathrm{p}=0.005)$.
\end{abstract}

Conclusions: Endothelial dysfunction may be related to hypoglycemia in T1D and to HDL-C in T2D

Keywords: Vascular Endothelial Function; RH-PAT Index; Type 1 Diabetes Mellitus; Type 2 Diabetes Mellitus; Continuous Glucose Monitoring; Hypoglycemia;

\section{Introduction}

Declining vascular endothelial function may indicate early stage of arteriosclerosis, and may be an independent risk factor for future cardiovascular events [1]. Thus, vascular endothelial function should be evaluated to perform appropriate risk assessments and therapeutic interventions. A widely used noninvasive test of vascular end othelial function is flow-mediated dilation (FMD), which quantifies changes in the brachial artery's diameter thatare caused by a blood flow-dependent vasodilatation reaction. This vasodilatation is related to endothelial-derived nitric oxide (NO) activity, which causes the relaxation of vascular smooth muscle and is triggered by increased blood flow and shear stress after the release of a vascularization. Thus, FMD can detect changes in the response to NO activity and vascular endothelial function, although this test is also influenced by various other factors $[2,3]$. Moreover, there are no standardized equipment or procedures for FMD (e.g., measuring conditions, cuff position, technique for measuring blood vessel diameter in the resting and expanded states), which can lead to inter-facility variations in the measured FMD values and subsequent problems with the reproducibility of FMD data [3].
Recent research has led to the introduction of a new method for measuring peripheral arterial hyperemia (reactive hyperemia peripheral arterial tonometry [RH-PAT]), which has low interexaminer variation and good reproducibility $[4,5]$. The RH-PAT technique is used to calculate an index of vascular endothelial function (RH-PAT index, RHI), which is correlated with various cardiovascular risk factors, such as body mass index (BMI), total cholesterol, high-density lipoprotein cholesterol (HDL-C), diabetes, and smoking [6]. Previous reports have also indicated that other indices of vascular endothelial function, such as FMD, are negatively correlated with the magnitude of blood glucose fluctuations and improve when blood glucose fluctuations are minimized [7-9]. However, few reports have addressed the relationship between RHI and daily blood glucose fluctuations [10, 11]. Therefore, the present study used RHI to evaluate vascular endothelial functions among patients with diabetes and used continuous glucose monitoring (CGM) to examine blood glucose parameters. These results were used to examine the associations between RHI and multiple arteriosclerosis-related factors, such as tumor necrosis factor-alpha (TNF- $\alpha$ ), interleukin-6 (IL-6), and advanced glycation end products (AGEs) according to the type of diabetes. 


\section{Patients and Methods}

\section{Patients}

Twenty-eight patients with diabetes aged $\geq 20$ years and who had glycated hemoglobin (HbA1c) levels of $6-10 \%$ visited our outpatient clinic between June 2014 and March 2016 were enrolled in the study. These individuals included 14 women with type 1 diabetes (T1D) and 14 patients (5 men, 9 women) with type 2 diabetes (T2D).

All patients with T1D were female and were treated using multiple daily insulin injections. Among patients with T2D, 1 patient was receiving insulin injections, 5 patients were receiving insulin injections and oral hypoglycemic agents, 6 patients were receiving only medication, and 2 patients were being treated using diet therapy. None of patients were used insulin pump, All patients underwent evaluations to determine their RHI, and 3 patients were excluded because of device errors, and data from 25 patients (12 with T1D and 13 with T2D) were included in the study. Patients were considered excluded from participation if they had active infections, severe liver/kidney diseases, eating disorders, or psychiatric disorders. All patients provided written informed consent prior to participation, and our study protocol was approved by the ethics committee of Tokyo Women's Medical University (131009; January 22, 2014).

\section{Glucose monitoring and RHI measurement processes}

Each patient completed CGM (iPro 2, Medtronic, Minneapolis, USA) for 3 days before RHI measurement, and their daily blood glucose fluctuations were recorded. RHI measurement was performed in a room with a constant luminous intensity and a temperature of $21-26^{\circ} \mathrm{C}$ at 8:00 a.m., before taking breakfast and after resting for more than 20 minutes. The measurement was carried out on the day of removing CGM, and then blood sampling was also performed.

\section{Testing parameters}

The patients' height, weight, BMI, abdominal circumference, and blood pressure were measured immediately before RHI measurement. Glucose metabolism was evaluated based on levels of blood glucose, HbA1c (HPLC method; Arkley, Kyoto), glycoalbumin (GA; Asahi Kasei Pharma, Tokyo), and 1,5-anhydroglucitol (1,5AG; Kyowa Medex, Tokyo). Additional laboratory testing was performed to obtain values for highsensitivity C-reactive protein, TNF- $\alpha$ (R\&D SYSTEMS, Minnesota), adiponectin (LSI Medience, Tokyo, Japan), low-density lipoprotein cholesterol (LDL-C), HDL-C, triglycerides, pentosidine, blood urea nitrogen, creatinine, aspartate aminotransferase, alanine aminotransferase, lactate dehydrogenase, alkaline phosphatase, gamma-glutamyl transpeptidase, fasting serum C peptide, plasma insulin, cystatin $\mathrm{C}$, and the albumin/creatinine ratio in the first morning urine (Nittobo Medical, Tokyo). The estimated glomerular filtration rate $\left(\mathrm{mL} / \mathrm{min} / 1.73 \mathrm{~m}^{2}\right)$ was calculated as $194 \times \mathrm{Cr}^{-1.094} \times \mathrm{Age}^{-0.287}(\times 0.739 \%)(\%$ for women)[12]. Pulse wave velocity was measured using the branchial/ankle method (Omron Colin Corporation Tokyo). The intima-media thickness (IMT) was measured using carotid B mode ultrasonography (Toshiba Aplio,
Tokyo), which was used to determine the values for the average bilateral mean IMT, the larger of the left or right IMT, and the plaque score.

Daily insulin dose (U/kg/day) was obtained from the patients' medical records. Fluctuations in blood glucose were evaluated based on the means of daily differences (MODD) [13], mean amplitude of glycemic excursions (MAGE) [14], J-index [15], and M-value [16]. MODD was defined as the average value for differences in blood glucose at the same time during two consecutive days. MAGE was defined as the average amplitude of blood glucose fluctuations that exceeded 1 standard deviation for the 24-h average blood glucose value from CGM. The J-index was calculated as $0.324 \times$ (mean blood glucose level + standard deviation $)^{2}$. The M-value was defined as the average deviation of measured blood glucose levels from the basic value of $100 \mathrm{mg} /$ dL.

\section{Measuring vascular endothelial function}

Vascular endothelial function was measured using an EndoPAT2000® device (Itamar Medical, Israel). One upper arm was avascularized and then subjected to RH-PAT to determine the change in blood flow in the index finger artery before and after avascularization. To adjust for changes in the rate of blood flow related to systemic circulation fluctuations and changes in the sympathetic nervous system, the non-avascularized side was also evaluated. The RHI value was then calculated by dividing RH-PAT on the avascularized side by that on the non-avascularized side. Higher RHI values reflect better vascular endothelial function, with an RHI value of 1.67 used as a cut-off to identify vascular endothelial dysfunction [17].

\section{Statistical analysis}

Continuous variables with parametric distributions were compared using Student's t test, and continuous variables with non-parametric distributions were compared using the MannWhitney U test. Univariate analyses of the correlations between RHI and clinical or laboratory parameters were performed using Pearson's correlation coefficient, as a parametric test, and Spearman's correlation coefficient, as a non-parametric test. Multivariate analysis was performed using RHI as the dependent variable and using the stepwise method. All analyses were performed using IBM SPSS software (version 21; IBM Japan, Tokyo, Japan), and the significance level was set at $p<0.05$.

\section{Results}

\section{Patients' clinical characteristics}

Patients with T2D had significant higher values than those with T1D for age, abdominal circumference, and uric acid. Patients with T1D had higher values for HDL-C than those with T2D (Table 1). There was no significant difference in RHI between patients with type 1 and type 2 .

\section{Clinical factors that were related to RHI among all cases}

Relation with RHI and clinical factors were evaluated using bivariate or multivariate analyses. RHI was positively correlated 
Table 1: Clinical characteristics

\begin{tabular}{|c|c|c|c|}
\hline & T1D & T2D & $\mathbf{P}$ \\
\hline $\mathbf{n}$ & 12 & 13 & \\
\hline Sex (male/female) & $0 / 12$ & $5 / 8$ & \\
\hline Age (years) & $41 \pm 13$ & $53 \pm 13$ & $<0.05$ \\
\hline Duration of diabetes (years) & $17.3 \pm 11.0$ & $12.4 \pm 9.2$ & 0.444 \\
\hline BMI $\left(\mathrm{kg} / \mathrm{m}^{2}\right)$ & $24.6 \pm 4.0$ & $27.0 \pm 4.0$ & 0.162 \\
\hline Abdominal circumference $(\mathrm{cm})$ & $80.6 \pm 10.4$ & $89.3 \pm 9.0$ & $<0.05$ \\
\hline HbA1c (\%) & $7.5 \pm 1.0$ & $7.0 \pm 0.9$ & 0.232 \\
\hline sBP (mmHg) & $123.0 \pm 21.0$ & $135.5 \pm 10.5$ & 0.073 \\
\hline dBP (mmHg) & $76.5 \pm 10.4$ & $78.5 \pm 8.4$ & 0.606 \\
\hline HR (bpm) & $81.7 \pm 13.8$ & $74.6 \pm 15.0$ & 0.243 \\
\hline TG (mg/dl) $*$ & $103.7 \pm 77.5$ & $88.7 \pm 40.3$ & 0.689 \\
\hline LDL-C (mg/dl) & $109.1 \pm 31.1$ & $108.8 \pm 25.0$ & 0.978 \\
\hline HDL-C (mg/dl) & $79.5 \pm 15.4$ & $60.0 \pm 14.8$ & $<0.05$ \\
\hline $\operatorname{eGFR}(\mathrm{ml} / \mathrm{min}) ※$ & $87.5 \pm 22.9$ & $83.2 \pm 24.3$ & 0.47 \\
\hline $\operatorname{ACR}(\mathrm{mg} / \mathrm{gCr}) *$ & $30.2 \pm 83.4$ & $5.3 \pm 4.4$ & 0.769 \\
\hline $\mathrm{UA}(\mathrm{mg} / \mathrm{dl})$ & $3.8 \pm 1.0$ & $5.5 \pm 1.3$ & $<0.05$ \\
\hline \multirow[t]{2}{*}{ RHI } & $2.08 \pm 0.74$ & $1.83 \pm 0.70$ & 0.194 \\
\hline & & Student's $\mathrm{t}$-test & *Mann-Whitney U test \\
\hline
\end{tabular}

Supplementary Table 1: Clinical factors correlated with RHI in all patients (n=25)

\begin{tabular}{|c|c|c|}
\hline Variable & R & P \\
\hline Age(years) & -0.065 & 0.757 \\
\hline Duration(years) & 0.137 & 0.112 \\
\hline BMI(kg/m²) & -0.325 & 0.206 \\
\hline HbA1c(\%) & 0.262 & 0.02 \\
\hline GA(\%) & 0.471 & 0.764 \\
\hline LDL-C(mg/dl) & 0.063 & 0.099 \\
\hline HDL-C(mg/dl) & 0.337 & 0.768 \\
\hline eGFR(ml/min) & -0.062 & 0.099 \\
\hline High-sensitivity CRP(ng/ml) & -0.352 & 0.302 \\
\hline Pentosidine $(\mu \mathrm{g} / \mathrm{ml})$ & -0.225 & 0.835 \\
\hline TNF- $\alpha(\mathrm{pg} / \mathrm{ml})$ & -0.046 & 0.347 \\
\hline MODD & 0.201 & 0.755 \\
\hline MAGE & 0.066 & 0.116 \\
\hline J-INDEX & 0.323 & 0.155 \\
\hline M-value* & 0.293 & 0.428 \\
\hline Frequency of hypoglycemia below $70 \mathrm{mg} / \mathrm{dl}(/$ day) & 0.166 & 0.586 \\
\hline
\end{tabular}



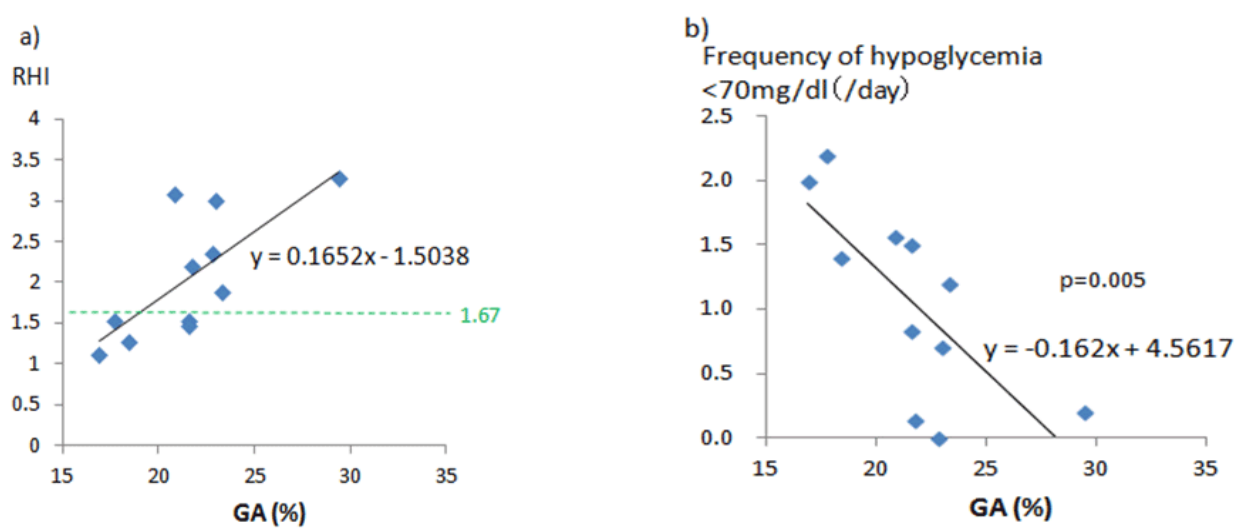

Figure 1:

with GA $(r=0.471, p=0.02)$, but was not significantly correlated with MODD, MAGE, J-index, or M-value (Suppl. Table 1, Suppl. Fig. 1). There was no significant difference in the RHI values between patients with T1D and T2D. In the multivariate analysis which was adjusted for age, duration of diabetes, BMI, FBG, GA, TG, HDL-C, and HbA1c, only GA was independently correlated with RHI $(\beta=0.462, p=0.026)$.

\section{Clinical factors that were related to RHI among patients with type 1 diabetes}

Among the patients with T1D, bivariate analyses revealed that RHI was strongly correlated with GA ( $r=0.727, p=0.011)$ (Table 2 , Figure 1a). In the multivariate analysis, which was adjusted for age, duration of diabetes, BMI, FBG, GA, average blood glucose,

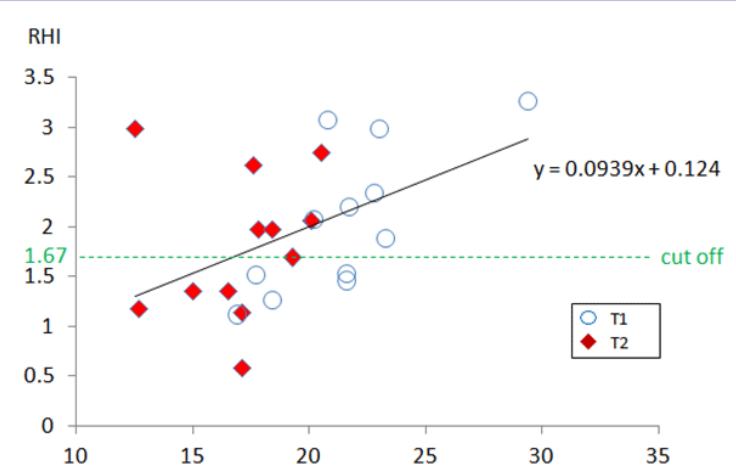

Table 2: Clinical factors related to RHI in patients with T1D (n=12)

\begin{tabular}{|c|c|c|}
\hline Variable & $\mathbf{R}$ & $\mathbf{P}$ \\
\hline Age (years) & -0.24 & 0.453 \\
\hline Duration of diabetes (years) & -0.277 & 0.383 \\
\hline BMI $\left(\mathrm{kg} / \mathrm{m}^{2}\right)$ & -0.406 & 0.191 \\
\hline FBG (mg/dl) & -0.414 & 0.181 \\
\hline HbA1c (\%) & 0.295 & 0.351 \\
\hline GA (\%) & 0.727 & 0.011 \\
\hline $\mathrm{TG}(\mathrm{mg} / \mathrm{dl}) *$ & 0.291 & 0.359 \\
\hline LDL-C (mg/dl) & 0.090 & 0.781 \\
\hline HDL-C (mg/dl) & -0.008 & 0.98 \\
\hline eGFR (ml/min) & 0.200 & 0.532 \\
\hline High-sensitivity CRP (ng/ml)※ & -0.373 & 0.259 \\
\hline Pentosidine $(\mu \mathrm{g} / \mathrm{ml})^{\%}$ & -0.582 & 0.06 \\
\hline TNF- $\alpha(\mathrm{pg} / \mathrm{ml})$ & 0.314 & 0.347 \\
\hline MODD & 0.298 & 0.347 \\
\hline MAGE & 0.138 & 0.669 \\
\hline J-INDEX & 0.445 & 0.148 \\
\hline CGM mean blood glucose (mg/dl) & 0.53 & 0.077 \\
\hline Frequency of hypo $<70 \mathrm{mg} /$ dl $(/$ day $)$ & -0.522 & 0.082 \\
\hline hypo: hypoglycemia & Pearson's correlation coefficient & ※spearman \\
\hline
\end{tabular}


TG, HDL-C, high-sensitivity CRP, TNF- $\alpha$, and pentosidine, only GA was independently correlated with RHI $(\beta=0.747, p=0.013)$. Among patients with T1D, GA was strongly and negatively correlated with the frequency of hypoglycemic events $(<70 \mathrm{mg} /$ $\mathrm{dL} ; \mathrm{r}=-0.725, p=0.012$ ) (Figure $1 \mathrm{~b}$ ).

\section{Clinical factors that were related to RHI among patients with type 2 diabetes}

Among patients with T2D, RHI was negatively correlated with
TG $(r=-0.601, p=0.03)$ and TNF- $\alpha(r=-0.583, p=0.047)$ but positively correlated with HDL-C $(r=0.588, p=0.034)$ (Table 3 , Figure 2). However, unlike among patients with T1D, RHI was not correlated with GA among patients with T2D. In the multivariate analysis, which was adjusted for age, duration of diabetes, BMI, FBG, GA, average blood glucose, TG, HDL-C, high-sensitivity CRP, TNF- $\alpha$, and pentosidine, only HDL-C was independently correlated with RHI $(\beta=0.586, p=0.045)$.

Table.3 Clinical factors related to RHI in T2D patients $(n=13)$

\begin{tabular}{|c|c|c|}
\hline Variable & $\mathbf{R}$ & $\mathbf{P}$ \\
\hline Age (years) & 0.256 & 0.398 \\
\hline Duration of diabetes (years) & 0.531 & 0.062 \\
\hline BMI $\left(\mathrm{kg} / \mathrm{m}^{2}\right)$ & -0.183 & 0.55 \\
\hline FBG $(\mathrm{mg} / \mathrm{dl})$ & 0.047 & 0.88 \\
\hline HbA1c (\%) & 0.156 & 0.611 \\
\hline GA (\%) & 0.171 & 0.576 \\
\hline $\mathrm{TG}(\mathrm{mg} / \mathrm{dl}) ※$ & -0.601 & 0.03 \\
\hline LDL-C (mg/dl) & 0.031 & 0.919 \\
\hline HDL-C (mg/dl) & 0.588 & 0.034 \\
\hline eGFR (ml/min) & -0.337 & 0.261 \\
\hline High-sensitivity CRP (ng/ml)* & -0.49 & 0.106 \\
\hline Pentosidine $(\mu \mathrm{g} / \mathrm{ml})^{*}$ & -0.217 & 0.498 \\
\hline $\mathrm{TNF}-\alpha(\mathrm{pg} / \mathrm{ml})$ & -0.583 & 0.047 \\
\hline MODD & -0.004 & 0.991 \\
\hline MAGE & -0.27 & 0.373 \\
\hline J-INDEX & -0.27 & 0.372 \\
\hline CGM mean blood glucose(mg/dl) & -0.051 & 0.868 \\
\hline Frequency of hypo < 70mg/dl（/day） & 0.17 & 0.58 \\
\hline hypo: hypoglycemia & Pearson's correlation coefficient & ※Spearmar \\
\hline
\end{tabular}

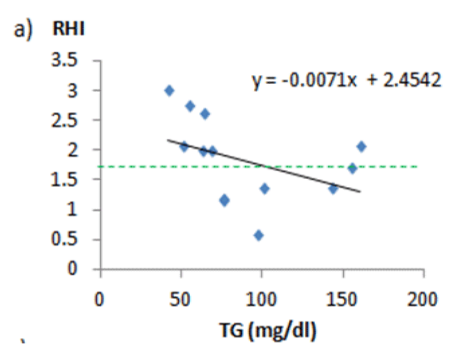

c) $\mathrm{RHI}$

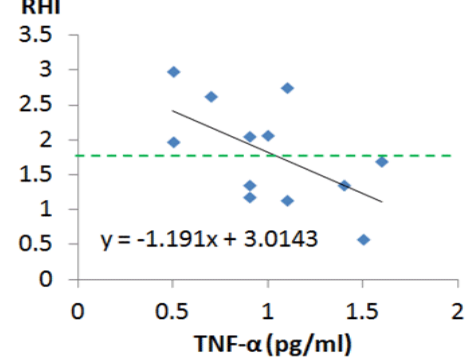

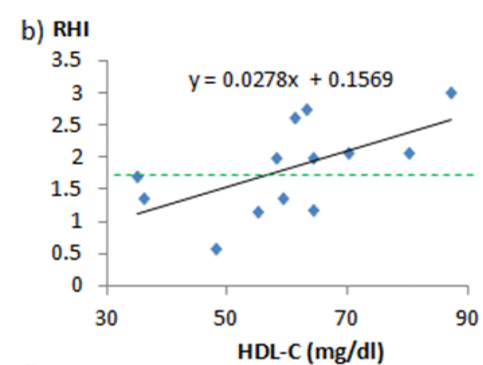

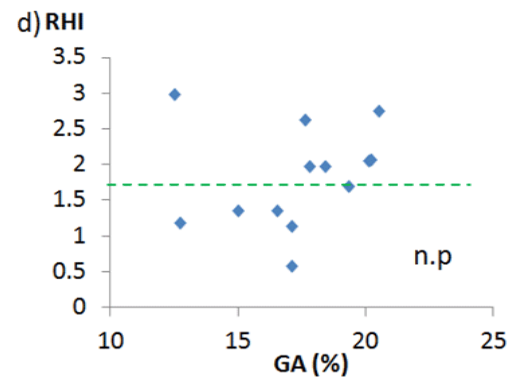

Figure 2: 


\section{Discussion}

The present study examined the relations of RHI with cardiovascular risk factors and daily fluctuation of blood glucose levels among patients with T1D and T2D, who did not exhibit any significant difference in their HbA1c levels. Among patients with T1D, RHI was positively correlated with GA, while RHI was positively correlated with HDL-C, among patients with T2D.

Among 4,352 patients from various cohorts of the Framingham study (55 \pm 16 years old, $51 \%$ female), RHI was significantly associated with age, systolic blood pressure, heart rate, BMI, total cholesterol/HDL-C ratio, diabetes, and smoking [18]. Furthermore, among 106 Japanese patients with T2D, RHI was significantly associated with the ankle-brachial index, carotid IMT, and statin use [19]. In the present study, RHI was not correlated with GA among patients with T2D, although RHI was correlated with lipid profile factors, TNF- $\alpha$, and HDL-C. These results indicate that RHI is correlated with arteriosclerotic factors and supports the results of the Framingham study [18] and those by Ueda et al. [19].

A previous study indicated that patients with T1D have remarkably lower RHI values than non-diabetic patients [20]. In addition, patients with T1D and endothelial dysfunction based on their RHI value tend to have high HbA1c levels [21]. A prospective study of 73 young patients with T1D suggested that RHI improved with better control of HbA1c [22], although this relationship was not observed in the present study.

The present study revealed that among patients with averaged 16-year durations of T1D, RHI was correlated with GA. In addition, GA was negatively correlated with the frequency of hypoglycemia $(<70 \mathrm{mg} / \mathrm{dL} ; \mathrm{r}=-0.727, \mathrm{p}=0.011)$, which suggests that RHI may decrease with hypoglycemia. This possibility is also supported by the fact that RHI may be reduced during vasoconstriction caused by sympathetic nerve stimulation. Furthermore, among patients with T1D, FMD values were found to be negatively correlated with two indicators of hypoglycemia (glycemic risk assessment diabetes equation [GRADE] and low blood glucose index [LBGI]) [23]. Moreover, patients with T1D and frequent severe hypoglycemic episodes exhibited higher levels of plaque in the carotid and femoral arteries, as well as notably increased serum markers of inflammation [24]. Although it remains unclear how hypoglycemia can influence changes in vascular function, hypoglycemia is known to stimulate the sympathetic nervous system to increase heart rate, cardiac output, myocardial contraction, systolic blood pressure, plasminogen activation inhibitor, and inflammatory reactions (e.g., through platelet activity, P-selectin, and high-sensitivity CRP) $[25,26]$. Thus, patients with diabetes who experience constant oxidative stress during hypoglycemia may experience compensatory physical changes, hematological changes, and an increased inflammatory response.

The onset or development of diabetic complications may be mediated by AGEs, which bind to receptors for AGE (RAGE) on almost all cells and activate intracellular signals. In this context, the AGE-RAGE system is a trigger of oxidative stress and is related to inflammation and apoptosis [27]. Furthermore, the accumulation of AGEs causes arteriosclerotic disease, and is involved in the development of microangiopathy and macroangiopathy in patients with diabetes. Although AGEs are through to decrease NO synthase expression in the vascular endothelium and subsequently decrease FMD-measured vascular endothelial function [28], no reports have suggested that AGEs may be related to RHI. The present study also failed to detect an association between RHI and serum pentosidine (one of the AGEs) among patients with T1D or T2D. However, further studies are needed to address this issue, since the present study only examined a small sample of young patients.

The present study demonstrated that it is possible to examine the relation between RHI and arteriosclerosis-related factors or CGM parameters. Another possible strength of the present study is that a single examiner performed all RHI measurements, which indicates that the measurement error may be very small. However, the present study is limited by the small sample size ( 28 patients) and the fact that 23 of the 28 patients were women.

\section{Conclusion}

The results from the present study suggest that vascular endothelial function (evaluated based on RHI from RH-PAT) decreases as GA decreases among patients with T1D, while endothelial function is maintained at high HDL-C levels among patients with T2D.

\section{Acknowledgements}

The authors thank Dr. Hiroko Kobayashi and Sari Hoshina for recruiting the patients for the present study. We also thank Edit age (www.editage.jp) for English language editing.

\section{Compliance with Ethical Standards}

All procedures followed were in accordance with the ethical standards of the institutional committee on human experimentation (131009; January 22, 2014) and with the Helsinki Declaration of 1964 and later versions. Informed consent was obtained from all patients who were included in the study.

\section{References}

1. Schächinger V, Britten $M B$, Zeiher AM. Prognostic impact of coronary vasodilator dysfunction on adverse long-term outcome of coronary heart disease. Circulation. 2000;101(16):1899-1906.

2. Corretti MC, Anderson TJ, Benjamin EJ, Celermajer D, Charbonneau F, Creager MA, et al. Guidelines for the ultrasound assessment of endothelial-dependent flow-mediated vasodilation of the brachial artery: a report of the International Brachial Artery Reactivity Task Force. J Am Coll Cardiol. 2002;39(2):257-265.

3. Thijssen DH, Black MA, Pyke KE, Padilla J, Atkinson G, Harris RA, et al. Assessment of flow-mediated dilation in humans: a methodological and physiological guideline. Am J Physiol Heart Circ Physiol. 2011;300(1):H2-12. doi: 10.1152/ajpheart.00471.2010

4. Bonetti PO, Barsness GW, Keelan PC, Schnell TI, Pumper GM, Kuvin JT et al. Enhanced external counterpulsation improves endothelial function in patients with symptomatic coronary artery disease. J Am Coll Cardiol. 2003;41(10):1761-1768. 
5. Kuvin JT, Patel AR, Sliney KA, et al. Assessment of peripheral vascular endothelial function with finger pulse wave amplitude. Am Heart J. 2003;146(1):168-174. Doi: 10.1016/S0002-8703(03)000942

6. Hamburg NM, Keyes MJ, Larson MG, Pandian NG, Sheffy J, Schnall $\mathrm{RP}$,et al. Cross-sectional relations of digital vascular function to cardiovascular risk factors in The Framingham Heart Study. Circulation. 2008;117:2467-2474. Doi: 10.1016/S00028703(03)00094-2

7. Maiorino MI, Casciano O, Della Volpe E, Bellastella G, Giugliano D, Esposito K. et al. Reducing glucose variability with continuous subcutaneous insulin infusion increases endothelial progenitor cells in type 1 diabetes: an observational study. Endocrine. 2016;52(2):244-252. Doi: 10.1007/s12020-015-0686-7

8. Harrington J, Peña AS, Wilson L, et al. Vascular function and glucose variability improve transiently following initiation of continuous subcutaneous insulin infusion in children with type 1 diabetes. Pediatr Diabetes. 2013;14(7):504-511. Doi: 10.1111/pedi.12050

9. Jamiołkowska M, Jamiołkowska I, Luczyński W, et al. Impact of RealTime Continuous Glucose Monitoring Use on Glucose Variability and Endothelial Function in Adolescents with Type 1 Diabetes: New Technology-New Possibility to Decrease Cardiovascular Risk? J Diabetes Res. 2016;2016:4385312. Doi: 10.1155/2016/4385312

10. Torimoto K, Okada Y, Mori H, Otsuka T, Kawaguchi M, Matsuda $M$, et al. Effects of exenatide on postprandial vascular endothelial dysfunction in type 2 diabetes mellitus. Cardiovasc Diabetol. 2015;14:25. Doi: 10.1186/s12933-015-0188-1

11. Torimoto K, Okada Y, Mori H, Tanaka Y. Relationship between fluctuations in glucose levels measured by continuous glucose monitoring and vascular endothelial dysfunction in type 2 diabetes mellitus. Cardiovasc Diabetol. 2013;12:1. Doi: 10.1186/1475-284012-1

12. Matsuo S, Imai E, Horio M, et al. Revised equations for estimated GFR from serum creatinine in Japan. Am J Kidney Dis. 2009;53(6):982992. Doi: 10.1053/j.ajkd.2008.12.034

13. Molnar GD, Taylor WF, Ho MM. Day-to-day variation of continuously monitored glycaemia: a further measure of diabetic instability. Diabetologia. 1972;8(5):342-348.

14. Service FJ, Molnar GD, Rosevear JW, Ackerman E, Gatewood LC, Taylor WF. Mean amplitude of glycemic excursions, a measure of diabetic instability. Diabetes. 1970;19(9):644-655.

15. Wójcicki JM. "J"-index. A new proposition of the assessment of current glucose control in diabetic patients. Horm Metab Res. 1995;27(1):41-42.

16. Schlichtkrull J, Munck O, Jersild M. The M-Value, an Index of Bloodsugar Control in Diabetics. Acta Medica Scandinavica. 1965;177:95102.

17. Bonetti PO, Pumper GM, Higano ST, Holmes DR Jr, Kuvin JT, Lerman
A, et al. Noninvasive identification of patients with early coronary atherosclerosis by assessment of digital reactive hyperemia. J Am Coll Cardiol. 2004;44(11):2137-2141. Doi: 10.1016/j. jacc.2004.08.062

18. Hamburg NM, Palmisano J, Larson MG, et al. Relation of brachial and digital measures of vascular function in the community: the Framingham Heart Study Hypertension. 2011;57(3):390-396. Doi: 10.1161/HYPERTENSIONAHA.110.160812

19. Ueda M, Takabe M, Sawada T, et al. Comparison Between 2 Types of Vascular Endothelial Function Test (Flow-Mediated Dilatation vs. Reactive Hyperemia Index) in Patients With Type 2 Diabetes Mellitus. J Jap Diabetes Society. 2017;60(5):370-377. Doi: 10.11213/ tonyobyo.60.370

20.Pareyn A, Allegaert K, Asscherickx W, et al. Impaired endothelial function in female adolescents with type 1 diabetes measured by peripheral artery tonometry. Eur J Pediatr. 2013;172(8):1017-1022. Doi: 10.1007/s00431-013-1988-5

21.Palombo C, Kozakova M, Morizzo C, Gnesi L, Chiara Barsotti M, Spontoni M, et al. Circulating endothelial progenitor cells and large artery structure and function in young subjects with uncomplicated type 1 diabetes. Cardiovasc Diabetol. 2011;10:8897. Doi: 10.1186/1475-2840-10-88

22.Scaramuzza AE, Redaelli F, Giani E, Macedoni M, Giudici V, Gazzarri A, et al. Adolescents and young adults with type 1 diabetes display a high prevalence of endothelial dysfunction. Acta Pediatrica. 2015;104(2):192-197. Doi: 10.1111/apa.12877

23.Peña AS, Couper JJ, Harrington J, et al. Hypoglycemia, but Not Glucose Variability, Relates to Vascular Function in Children with Type 1 Diabetes. Diabetes Technol Ther. 2012;14(6):457-462. Doi: 10.1089/dia.2011.0229

24.Giménez M, Gilabert R, Monteagudo J, Anna Alonso Roser Casamitjana, Ignacio Conget. Repeated Episodes of Hypoglycemia as a Potential Aggravating Factor for Preclinical Atherosclerosis in Subjects With Type 1 Diabetes. Diabetes Care. 2011;34(1):198-203. Doi: $10.2337 / \mathrm{dc} 10-1371$

25.Gogitidze JN, Hedrington MS, Briscoe VJ, Tate DB, Ertl AC, Davis SN. Effects of acute hypoglycemia on inflammatory and proatherothrombotic biomarkers in individuals with type 1 diabetes and healthy individuals. Diabetes Care. 2010;33(7):1529-1535. Doi: $10.2337 /$ dc09-0354

26. Wright RJ, Newby DE, Stirling D, Ludlam CA, Macdonald IA, Frier BM. Effects of acute insulin-induced hypoglycemia on indices of inflammation: putative mechanism for aggravating vascular disease in diabetes. Diabetes Care. 2010;33(7):1591-1597. Doi: 10.2337/dc10-0013

27. Stirban A, Gawlowski T, Roden M, et al. Vascular effects of advanced glycation end products: Clinical effects and molecular mechanisms. Mol Metab. 2013;3(2):94-108. Doi: 10.1016/j.molmet.2013.11.006

28.Xiaomei Ren, Ligun Ren, Qin Wei, Shao H, Chen L, Liu N. Advanced glycation end-products decreases expression of endothelial nitric oxide synthase through oxidative stress in human coronary artery endothelial cells. Cardiovasc Diabetol. 2017;16(1):52. Doi: 10.1186/ s12933-017-0531-9 